\title{
Wie Banken den Strukturwandel finanzieren
}

\section{Christina Maier}

\section{Relevanz}

Wenn die Produktivität hoch sein soll, müssen Arbeit und Kapital dorthin wandern, wo die Erträge hoch und die Zukunftsperspektiven gut sind, und dürfen nicht dort verharren, wo Beschäftigung und Einkommen unsicher sind. Innovatives Wachstum löst einen andauernden Strukturwandel kreativer Zerstörung aus. Dieses Wachstum kann sich erst entfalten, wenn der Strukturwandel gelingt. Schwache Unternehmen dürfen nicht länger Marktanteile besetzen und damit das Wachstum der produktiven Unternehmen bremsen. Banken dürfen faule Kredite an wenig wettbewerbsfähige Unternehmen nicht weiter verlängern, sondern müssen sie fällig stellen und teilweise abschreiben, damit sie die Kreditvergabe auf die Wachstumsunternehmen lenken können. Schwache Banken mit wenig Eigenkapital können den Strukturwandel nicht finanzieren, weil die dabei auftretenden Verluste ihre Mindestkapitalausstattung gefährden. Nicht nur die Finanzstabilität, sondern auch das Produktivitätswachstum setzt einen starken Bankensektor mit robuster Kapitalausstattung voraus.

\section{Quelle}

Schivardi, Fabiano, Enrico Sette und Guido Tabellini (2017), Credit Misallocation During the European Financial Crisis, CEPR DP 11901.

C. Maier $(\bowtie)$

Universität St.Gallen, St.Gallen, Schweiz

E-Mail: christina.maier@student.unisg.ch 
Nach der Finanzkrise nahm in zahlreichen europäischen Ländern die Sorge zu, dass der geschwächte Bankensektor nicht mehr ausreichend in der Lage ist, Kapital zu den vielversprechendsten Projekten zu lenken, und stattdessen wenig produktive Problemfirmen weiterfinanziert und damit die Krise verlängert. Ein zentrales Problem stellen die Anreize bei der Kreditvergabe durch schwach kapitalisierte Banken dar. Sie neigen dazu, Kredite an schwache Unternehmen mit geringen Erfolgschancen zu verlängern, obwohl diese den Kredit oft nicht zurückzahlen können. So können Banken vermeiden, dass sie bei Kündigung von Problemkrediten diese in ihrer Bilanz sofort abschreiben müssen, wodurch sich ihr Eigenkapital verringert und das Einhalten der regulatorischen Kapitalvorschriften erschwert wird. Im Gegensatz dazu sind Banken mit solidem Kapitalpuffer eher bereit, faule Kredit abzuschreiben und neue Kredite an expandierende Unternehmen zu vergeben, anstatt bestehende Kreditlinien zu verlängern.

Das Verhalten schwach kapitalisierter Banken fördert eine Fehlallokation von Krediten. Wenig profitable Unternehmen erhalten zu viel, und gesunde zu wenig Kapital, was ihre Investitionen und ihr Wachstum einschränkt. Da kaum überlebensfähige Firmen zu wenig oft aus dem Markt ausscheiden, sind Produkt- und Faktormärkte überlaufen. Die überlebenden Firmen besetzen Marktanteile und mindern die Wachstumschancen der gesunden Unternehmen. Die Produktivität des Kapitaleinsatzes leidet. Das ,Verlorene Jahrzehnt' Japans in den 1990er Jahren gilt als Beispiel dafür, wie ein schwächelnder Bankensektor eine Krise verlängern kann.

Allerdings ist es schwer, unprofitable Unternehmen von solchen zu unterscheiden, die nur vorübergehend in Schwierigkeiten, aber grundsätzlich gut aufgestellt sind. Solchen Unternehmen hilft ein Aufrechterhalten von Kreditlinien über eine Krise hinweg und verhindert vermeidbare Insolvenzen und damit verbundene Arbeitsplatzverluste. Folglich sinken gesamtwirtschaftliche Nachfrage und Beschäftigung weniger stark. Ein weiterer positiver Aspekt liegt in den engen Lieferverflechtungen zwischen Unternehmen. Die Weiterführung von Krediten kann Insolvenzen vermeiden, welche die Produktion in anderen gesunden Unternehmen stören könnten, weil z. B. Lieferanten von schwer ersetzbaren Vorleistungen ausfallen. Diese beiden Effekte können zumindest kurzfristig den negativen Auswirkungen einer Fehlallokation von Krediten entgegenwirken.

Fabiano Schivardi, Enrico Sette und Guido Tabellini untersuchen am Beispiel Italiens im Zeitraum 2004 bis 2013 zwei Fragen: Welche Banken tragen zur Fehlallokation von Krediten bei? Welche Auswirkungen hat dieses Verhalten auf die Realwirtschaft, insbesondere auf das Wachstum und den Erfolg gesunder Unternehmen sowie auf das Produktivitätswachstum? Die Finanz- und Wirtschaftskrise führte in Italien zu einer besonders langen Rezession mit einem kumulativen Einkommensverlust von rund $10 \%$. Der Anteil notleidender Kredite in den Bankbilanzen stieg von knapp 6 auf $16 \%$ und die Kreditvergabe ging dauerhaft 
zurück. Die Forscher nutzen einen umfangreicheren Datensatz mit Informationen aus dem italienischen Firmenregister, dem Kreditregister und den Aufsichtsberichten der italienischen Nationalbank. Dieser umfasst mehr als 240'000 Unternehmen mit Kreditbeziehungen zu 163 italienischen Banken.

Die Wissenschaftler analysieren die Kreditvergabe von Banken an sogenannte ,Zombie'-Unternehmen. Als solche gelten Unternehmen, die entweder besonders ertragsschwach oder hoch verschuldet sind. Für eine Bank steigt damit das Kreditrisiko, während die Wahrscheinlichkeit einer vollständigen Rückzahlung abnimmt. Bereits die beschreibende Datenanalyse weist auf einen Zusammenhang zwischen der Kapitalisierung einer Bank und ihrer Kreditvergabe an schwache Unternehmen hin. Abb. 1 verdeutlicht, dass Banken mit einer niedrigen Eigenkapitalquote (Banken in den ersten beiden Quartilen der Eigenkapitalausstattung) über den gesamten beobachteten Zeitraum einen signifikant höheren Anteil von Krediten an ,Zombie'-Unternehmen aufweisen als Banken mit überdurchschnittlichen Eigenkapitalquoten (in den beiden oberen Quartilen des Datensatzes).

Während der Finanz- und Wirtschaftskrise war das jährliche Kreditwachstum an ,Zombie'-Unternehmen bei Banken mit einer Eigenkapitalquote unter dem Median um ein Viertel höher als bei jenen mit einer Eigenkapitalquote über dem Median.

Die ökonometrischen Schätzungen zeigen, dass die Höhe der Eigenkapitalquote einer Bank ihre Kreditvergabe an ,Zombie"-Unternehmen während der Finanz-

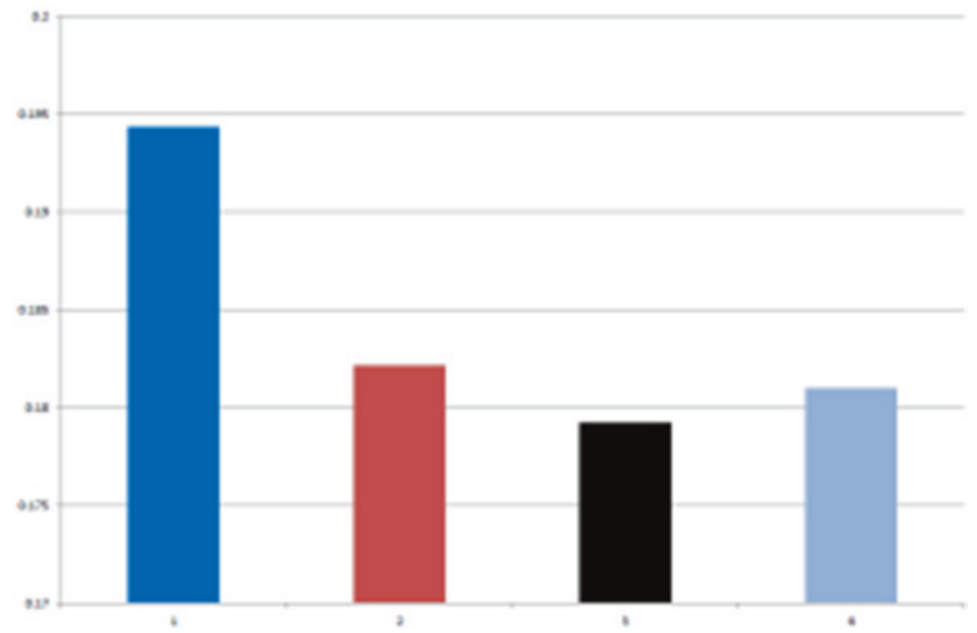

Abb. 1 Anteil der Kredite an ,Zombie'-Unternehmen an den Gesamtkrediten nach Quartil der regulatorischen Eigenkapitalquote, 2004-2013. (Quelle: Schivardi et al. 2017, S. 46) 
krise (2008-2013) signifikant beeinflusste. Das Kreditwachstum an diese Unternehmen war während der Krise bei Banken mit einer niedrigen regulatorischen Eigenkapitalquote von unter dem Median von $11 \%$ um ein Viertel bzw. 2 Prozentpunkte höher als bei Banken mit einer höheren Eigenkapitalquote. Vor der Finanzkrise zeigten sich kaum Unterschiede bei der Kreditvergabe an ,Zombie“Unternehmen zwischen schwach und gut kapitalisierten Banken. Die Finanzkrise verursachte bei vielen Banken erhebliche Abschreibungen und Verluste. Banken mit niedriger Eigenkapitalbasis liefen dadurch Gefahr, näher an oder sogar unter die regulatorische Mindestquote zu sinken. Gerade jene Banken hielten daher Kredite an notleidende Unternehmen aufrecht in der Hoffnung, dass sie sich erholen und den Kredit später wieder zurückzahlen würden. Dadurch konnten sie Abschreibungen von Problemkrediten und einen weiteren Rückgang ihrer Eigenkapitalquote vermeiden oder wenigstens aufschieben. Denn während einer Finanzkrise ist es besonders schwierig, neues Eigenkapital aufzunehmen, da die Investoren oft die Eigenkapitalaufnahme mit Problemen bei der Bank in Verbindung bringen. Sie sind unter diesen Umständen meist nur bei hohen Abschlägen bereit, weiteres Kapital zur Verfügung zu stellen.

Wie wirkt sich die verzerrte Kreditvergabe durch schwache Banken auf die Realwirtschaft und die Dauer einer Rezession aus? Einerseits beeinträchtigt die Fehlsteuerung der Kreditvergabe das Wachstum von gesunden Unternehmen, weil sie insgesamt weniger Kredite bekommen. Gleichzeitig erhöht sich die Überlebenswahrscheinlichkeit eines ,Zombie'-Unternehmens, dessen Kredite weiterlaufen. Normalerweise lenken Banken die Kredite von wenig profitablen Unternehmen zu den gesunden und tragen dazu bei, die Produktivitätsunterschiede abzubauen. Dieser produktivitätssteigernde Prozess blockiert jedoch, wenn bestehende Kreditlinien an schwache Unternehmen weiterlaufen. In der Folge lahmt das Produktivitätswachstum. Die empirischen Schätzungen deuten allerdings darauf hin, dass die Kreditvergabe durch schwach kapitalisierte Banken das Wachstum der gesunden Unternehmen kaum beeinträchtigt. Zwar schneiden sie im Vergleich zu ,Zombie'-Unternehmen nicht mehr so gut ab, wenn diese weiterhin Zugang zu Krediten haben und nicht so stark schrumpfen müssen. Dennoch verringert das Überleben schwacher Firmen die Arbeitsnachfrage, den Kapitaleinsatz und den Umsatz der gesunden Unternehmen insgesamt nicht.

Die Fehlsteuerung der Kreditvergabe wirkt sich absolut betrachtet kaum negativ auf das Wachstum von gesunden Unternehmen aus. Relativ zu den ,Zombie“-Firmen gehen jedoch Arbeitsnachfrage, Kapitaleinsatz und Umsatz zurück.

Auch auf die Produktivitätsunterschiede unter den Unternehmen wirkt sich die verzerrte Kreditvergabe während der Finanzkrise nicht signifikant aus. Nur wenn 
der Anteil der ,Zombie‘-Unternehmen sehr hoch (über $21 \%$ ) liegt, nehmen die Produktivitätsunterschiede zu.

Dagegen beeinflusst die Kreditvergabe eines schwachen Bankensektors das Insolvenzrisiko der Unternehmen. ,Zombie'-Unternehmen erhalten weiter Kredite, überleben öfter und nehmen den gesunden Unternehmen Marktanteile weg, sodass am Ende gerade diese öfter insolvent werden. Als Folge sind letztlich zu wenig gesunde Unternehmen und zu viele nicht überlebensfähige aktiv. Könnte man die Eigenkapitalquote aller untersuchten Banken auf ein Niveau höher als der Medianwert von $11 \%$ anheben, stiege die Ausfallrate der ,Zombie'-Unternehmen um 0,4 Prozentpunkte. Die Ausfallrate gesunder Unternehmen hingegen würde um 0,4 Prozentpunkte sinken. Letzteres entspricht einem Rückgang um ein Fünftel.

Hätten alle Banken in Italien eine Eigenkapitalquote mindestens in der Höhe des Medianwerts von 11 Prozent, wäre die Ausfallrate gesunder Unternehmen um ein Fünftel geringer.

Die Forscher zeigten, dass Italiens schwach kapitalisierte Banken während der Finanzkrise $\mathrm{zu}$ viele Kredite an wenig aussichtsreiche, kaum überlebensfähige Unternehmen vergeben haben. Die Fehlsteuerung der Kreditvergabe ermöglichte das Überleben vieler schwacher Unternehmen und verursachte $\mathrm{zu}$ viele Insolvenzen von gesunden Firmen. In der Folge lahmte das BIP-Wachstum. Hätte die italienische Regierung vier Milliarden Euro in schwach kapitalisierte Banken investiert, um deren Eigenkapitalquote auf die Höhe des Medianwerts anzuheben, wäre die jährliche Wachstumsrate von 2008 bis 2013 um etwa 0,2 bis 0,35 Prozentpunkte höher gewesen. Dies entspricht einem Anstieg von rund einem Zehntel.

Open Access Dieses Kapitel wird unter der Creative Commons Namensnennung 4.0 International Lizenz (http://creativecommons.org/licenses/by/4.0/deed.de) veröffentlicht, welche die Nutzung, Vervielfältigung, Bearbeitung, Verbreitung und Wiedergabe in jeglichem Medium und Format erlaubt, sofern Sie den/die ursprünglichen Autor(en) und die Quelle ordnungsgemäß nennen, einen Link zur Creative Commons Lizenz beifügen und angeben, ob Änderungen vorgenommen wurden.

Die in diesem Kapitel enthaltenen Bilder und sonstiges Drittmaterial unterliegen ebenfalls der genannten Creative Commons Lizenz, sofern sich aus der Abbildungslegende nichts anderes ergibt. Sofern das betreffende Material nicht unter der genannten Creative Commons Lizenz steht und die betreffende Handlung nicht nach gesetzlichen Vorschriften erlaubt ist, ist für die oben aufgeführten Weiterverwendungen des Materials die Einwilligung des jeweiligen Rechteinhabers einzuholen.

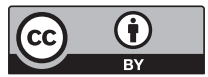

